\title{
La prensa belga durante el unionismo y el postunionismo (1830-1857)
}

\author{
Dra. Isabel Fernández Alonso
}

Universidad Libre de Bruselas

\begin{abstract}
El artículo traza el panorama de la prensa belga en las primeras décadas de la independencia. Se trata de una prensa esencialmente política, vinculada a las dos grandes corrientes ideólogicas del unionismo, liberales y católicos, y a los diferentes movimientos de oposición -orangistas, francófilos, republicanos, y demócratas-, sin olvidar el papel jugado por las publicaciones oficiales en la construcción de una mentalidad nacional, a imitación de la política realizada en oficiales en la construcción de una mentalidad nacional, á
los años inmediatamente anteriores por el reino holandés.

La libertad de prensa surge en Bélgica con la revolución de 1830, pero la democratización de la edición y lectura de periódicos habrá de esperar a la siguiente revolución, la de 1848, que trajo consigo la abolición del impuesto del timbre.

Dada la inexistencia de bibliografía en español sobre la historia de la prensa en Bélgica, nos hemos propuesto trazar en varios artículos las líneas esenciales de su evolución ${ }^{I}$. La delimitación temporal obedece a los vaivenes ideológicos del país. Con más motivo en este primer trabajo, pues la prensa decimonónica es una prensa esencialmente política.

Unionismo y postunionismo

on los tratados de Utrecht y Rastadt (1713-1714), España pierde sus posesiones en los Países Bajos. A partir de este momento Bélgica estará, sucesivamente, bajo dominio austriaco hasta 1795, francés hasta 1815 , y sometida a la soberanía holandesa desde el Congreso de Viena hasta su independencia en 1830.
\end{abstract}

1 Hemos realizado este trabajo gracias a una estancia de varios meses en la Universidad Libre de Bruselas. Casi toda la bibliografía que iremos mencionando está recogida en el repertorio, dirigido por Sophie Mathelart, Pour l'histoire des médias en Belgique: bibliographie 1830 à nos jours (Bruxelles: Centre de Études et de Recherches sur les Médias en Europe, 1994), que nos ha servido de excelente punto de partida. Asimismo, hemos de aclarar que nuestro desconocimiento de la lengua neerlandesa nos ha impedido la consulta de los trabajos realizados en el ámbito flamenco.

A los escos A los estudiosos de la Historia de la Comunicación que visiten Bélgica recomendamos, no obstante, la visita de los mon do auténticas joyas para quien desee acercarse a la historia de la imprenta y del periodismo. Si Pantino es un hombre muy conocido en España, no ocurre lo mismo con Verhoeven, editor en 1605 del primer periódico del mundo, Nieuwe Tydinghen, según los datos que poseemos hasta el momento. 
Junto a los conflictos comerciales -los holandeses establecieron, por ejemplo, un peaje para todos los navíos que remontaban el Escaut- y las diferencias linguísticas -en este momento el francés era también la lengua de la nobleza y la burguesía flamencas-, había un importante motivo de discordia entre belgas y holandeses: mientras que los primeros profesaban el catolicismo, el calvinismo se había convertido en la religión principal de los segundos.

Apunta Georges-Henri Dumont que Guillermo I de Holanda "se ganó la oposición de los católicos al imponer un control del Estado sobre la enseñanza, mientras que la joven burguesía liberal, formada en las nuevas universidades de Lieja y Gante, reivindicaba la elección directa de los parlamentarios, la responsabilidad ministerial y la libertad de prensa". Motivos comunes de queja eran los impuestos de molienda y tala de árboles, que afectaban esencialmente a los belgas, y la sobrerrepresentación holandesa en los Estados Generales y en el funcionariado.

Así las cosas, desde 1828 católicos y liberales belgas hacen causa común en pos de la independencia de su territorio, que lograrán dos años después. Inician as una andadura conjunta -lo que se ha denominado unionismo-, que perderá su razón de ser a partir de 1839 , momento en que desaparece por completo la amenaza holandesa, al firmar Guillermo I el Tratado de los XXIV Artículos ${ }^{3}$. Si bien, como indica Xavier Mabille, la práctica unionista no se rompe formalmente hasta agosto de 1847 con la formación del gobierno Rogier-Frère-Orban, de composición íntegramente liberal ${ }^{4}$.

Así, poco a poco, y aunque todavía se configuraron dos nuevos gabinetes de carácter unionista -los presididos por Henri De Brouckère (octubre de 1852) y Pierre De Decker (marzo de 1855)-, católicos y liberales fueron redefiniendo sus planteamientos hasta llegar a una clara bipolarización de la vida política del país ${ }^{5}$. No obstante, los primeros no se estructurarán formalmente como partido hasta 1884. El Partido Liberal, sin embargo, se funda ya en 1846. Desde entonces, los

2 Cfr. DUMONT, G.H., La Belgique. París: Que sais-je?, 1993, p. 59.

Este acuerdo había sido impuesto a Bélgica por las potencias internacionales tras la Campaña de los Diez Días, con la que el soberano holandés había intentado recuperar el territorio belga en 1831. Pero Guillermo I no lo firma hasta 1839, cuando reconoce la dificultad de una reconquista completa de la Bélgica independiente. Con el Tratado de los XXIV artículos Bélgica pierde el Luxemburgo alemán y una parte de la provincia de Limburgo.

4 Antes de éste y desde 1831 hubo en Bélgica siete gobiernos (Muelenaere, Goblet-Lebeau-Rogier, de Theux, Lebeau-Rogier, Nothomb, Van de Weyer, y de Theux-Malou), el cuarto de composición esencialmente libera y el séptimo de talante claramente católico, como aclara el propio Mabille (Cfr. Histoire politique de la Belgique. Bruxelles: Centre de recherche et d'information socio-politiques, 1992).

5 No obstante, es obligado precisar que en los dos grandes bloques políticos ya se van definiendo durante el unionismo tendencias divergentes. Dentro del Partido Liberal había un sector doctrinario, con Frère-Orban a frente, y un sector progresista, liderado por Paul Janson; mientras que en el ámbito católico se distinguían do corrientes: los constitucionales, animados por el cardenal Sterckx, y los ultramontanos, entre los que destac el economista Charles Périn. 
liberales ocupan el poder -además de en los casos antes mencionados- entre 1857 y 1870 y entre 1878 y 1884 . Desde esta fecha y hasta las elecciones de noviembre de 1919 -primeras en la historia de Bélgica con sufragio universal masculino-, los sucesivos ejecutivos estarán en manos de los católicos, favorecidos, sin duda, por la ferozmente antirreligiosa ley Van Humbeeck, votada por los liberales en 1879, y que generó la llamada "guerra de las escuelas".

Nosotros intentaremos trazar en este primer trabajo un panorama de la prensa belga entre el momento de la independencia y el año 1857, que marca, como acabamos de indicar, el inicio de una etapa de preeminencia liberal, a la que seguirán, desde 1884 hasta el estallido de la Primera Guerra Mundial, treinta años de ininterrumpidos gobiernos católicos.

Las publicaciones oficiales y el nacimiento del estado belga

La revolución belga estalló de modo espontáneo el 25 de agosto de 1830 a las puertas del Teatro de la Monnaie, donde una multitud -condicionada por la crisis económica y los ecos de la revuelta parisina-, que acababa de participar en diversas manifestaciones patrióticas, esperaba el fin de la representación de $L a$ Muette de Portici -ópera de Auber que relata la insurrección de Nápoles contra España de 1647.

El conflicto se extiende rápidamente, mientras -con la anuencia de las autoridades- se improvisa una guardia burguesa que logra controlar a las masas. Pero Guillermo I se niega a hacer concesiones y envía a sus tropas para aplacar a los insurgentes. Una vez que aquellas, con la ayuda francesa, fueron derrotadas en casi todos los frentes, un gobierno provisional proclama la independencia de Bélgica el 4 de octubre del mismo año 1830 y anuncia la convocatoria de un Congreso Nacional, que enseguida se convertirá en Asamblea Constituyente. La Constitución belga de 1831 -aún hoy vigente con diversas modificaciones, especialmente en lo relativo a la organización territorial del Estado- reconoce un amplio abanico de libertades, entre las que se cuenta la libertad de prensa. Se establece una monarquía parlamentaria y el príncipe Léopold de Saxe-CobourgGotha reinará hasta su muerte en 1865 como Léopold I. Nace así un régimen burgués censitario donde, como ya hemos indicado, liberales moderados y católicos compartirán el poder hasta que desaparezca por completo la amenaza holandesa.

Precisamente para reforzar la independencia de Bélgica, sobre todo en los primeros años, los gobiernos unionistas prestaron una especial atención a las publicaciones oficiales. En este sentido, el gobierno provisional firma el 21 de diciembre de 1830 un contrato con M. Feuillet-Dumus para la edición de L'Union belge. Relata Els Witte que a esta empresa dedicaron los revolucionarios una cuarta parte del presupuesto del Ministerio del Interior, aplicando, de este modo, una política de fomento de la prensa gubernamental, que habían atacado con dureza en los años anteriores, cuando era Guillermo de Orange quien la pro- 
movía.

Dado que L'Union belge no lograba publicar con la debida regularidad los documentos oficiales, el gobierno decide la fusión de éste con L'Indépendent el 9 de marzo de 1931.

Pero el verdadero periódico oficial del nuevo régimen fue, sin duda, $L e$ Moniteur belge, fundado el 16 de junio del mismo año 31, tras el fracaso de la fusión que acabamos de mencionar. Su politización será notoria en el primer lustro del unionismo ${ }^{6}$.

Por otro lado, nueve meses después del nacimiento del Moniteur surge Le Mémorial belge, de la mano de un grupo de padres de la patria, entre los que se contaban Lebeau, Devaux, Nothomb y Rogier, animados, al parecer, por el propio monarca, a quien interesaba el fortalecimiento del régimen unionista, pues le permitía mantener un cierto poder de arbitraje. El Mémorial -que se fusiona un año después de su fundación con L'Indépendent- podría definirse como una publicación de oposición moderada, debido a la fuerte personalidad de su redactor jefe, el liberal M. Faure, que no se sometía con facilidad a los dictados del Ejecutivo y del Monarca.

Pero hay que aclarar que la libertad de prensa, establecida ya por decreto de 16 de octubre de 1830, permitió el florecimiento de múltiples publicaciones de oposición -republicanas, orangistas, partidarias de la reunificación con Francia y demócratas-, cuyas tiradas sobrepasaban con creces las de las publicaciones oficiales en estos primeros momentos de la independencia. A. WARZEE ha realizado una catalogación detallada de todos los periódicos -en su mayoría efímeros- editados en Bélgica por estos años (Essai historique et critique sur les journaux belges. Journaux politiques. Bruselas: 1845). No obstante, consideramos que los escasos datos ofrecidos por Warzee han de ser cotejados mediante el análisis de los números de estas publicaciones que aún se conserven, sobre todo si queremos trazar conclusiones definitivas sobre el conjunto de la prensa durante el unionismo, pues, como veremos, hasta el momento sólo los periódicos oficiales, católicos y demócratas han sido estudiados con cierta profundidad.

A partir de 1840, una vez consolidada la independencia del joven estado, el panorama se simplificará y los periódicos se alinearán en torno a los ya citados grupos ideológicos del XIX belga: liberales, católicos y demócratas.

\section{La difícil organización de la prensa católica}

Desde mayo de 1831 importantes personalidades del mundo católico comienzan a reunir fondos para poner en marcha en Bruselas un periódico que

6 Para profundizar en las cuestiones que abordamos en este epígrafe y, en especial, en la evolución de este periódico, cfr. WITTE, E., Le Moniteur belge, le gouvernement et le parlement pendant l'Unionisme (1831periódico, cfr. WITTE, E., Le Moniteur belge, 
recoja sus inquietudes. Así surgen sucesivamente diversos títulos -Le Siècle, L'Union, Le Conservateur belge y la Revue de Bruxelles-, de los que sólo el último, de la mano de Adolphe Dechamps, alcanza cierta relevancia. En cualquier caso, al concluir la década de los treinta no había en Bruselas ningún diario de tendencia católica.

Por contra, la ciudad donde prolifera con más fuerza este tipo de prensa es Lieja, que había sido un principado independiente, gobernado por los príncipesobispos desde finales del siglo X hasta su anexión a Francia, a finales del XVIII. Allí se edita desde 1820 Le Courrier de la Meuse. Cuando en 1835 Dieudonné Stas asume la dirección del periódico, comienzan los problemas con el obispo Van Bommel, que no acepta la independencia de criterio de Stas.

Este conflicto explica el origen de la Gazette de Liège, promovida por el propio Van Bommel, con la ayuda de los condes Eugène de Mean y Emile d'Oultremont. Pronto este periódico será considerado el órgano del Obispado, y hará perder al Courrier de la Meuse un número importante de suscriptores?

En este contexto, Stas asume el reto de lanzar un gran diario católico en la capital belga, que fijase la línea editorial de la prensa provincial de esta tendencia. El Journal de Bruxelles contaría con un equipo de redactores de prestigio y un buen número de abonados, entre los que se habrían de contar los jóvenes graduados en la Universidad de Lovaina. Pero Stas tropieza con una gran dificultad: la negativa del cardenal Sterckx a prestar apoyo financiero a su proyecto. A. Cordewiener explica este hecho, no por las críticas vertidas desde el Vaticano a la libertad de prensa, sino por el temor del Cardenal a que su autoridad moral se viese comprometida por la marcha de un periódico al que había dado su apoyo ${ }^{8}$. No obstante, los obispos tenían un parecer distinto sobre las relaciones entre el clero y la prensa y normalmente ayudaban económicamente a las publicaciones católicas que se editaban en sus respectivas diócesis.

Así las cosas, el Journal de Bruxelles comienza a editarse el 1 de enero de 1841, con un aspecto más modesto que el proyectado por Stas; casi idéntico, por otra parte, al del Courrier de la Meuse, que había dejado de publicarse el día anterior, por decisión del propio Stas. Pero el número de abonados no era suficiente para garantizar la autonomía financiera del diario, por lo que algunos obispos decidieron aportar dinero, desobedeciendo las órdenes del episcopado, que sólo autorizaba la difusión en los centros de culto de listas de suscripción al Journal de Bruxelles.

7 Cabe recordar que la Gazette de Liège fue absorbida por La Libre Belgique en 1967.

8 Cfr. CORDEWIENER, A., "Attitudes des catholiques et de l'épiscopat devant les problèmes posés par l'orr Conition de leur press à Bruxelles (1831-1843)", en Revue belge d'Histoire Contemporaine, II, 1, 1970, pp. 27 a 43. 
Este periódico -cuya tendencia constitucional se definirá claramente a partir del célebre Congreso de Malines- fue adquirido por Paul Nève en $1856^{\circ}$. Nève compra también por estas fechas L'Union belge, La Gazette de Bruxelles, y L'Emancipation, que, a impulso de los hermanos Malou, se fusionará con el Journal de Bruxelles en $1858^{10}$. De este modo surge en la capital belga uno de los dos grupos de prensa católicos más importantes de finales de los años cincuenta en este país. El otro era el dirigido por Neut, conocido entonces como "el Alejandro Dumas de la prensa conservadora", (Patrie, Standaerd van Vlaenderem, Vlaemsche Land, y Gazette van Vlaenderen en Braband), con difusión en Gante y Brujas ${ }^{11}$.

Por otro lado, en 1853, bajo el impulso del obispo Delebecque, se había fundado en Gante Le Bien Public, de tendencia marcadamente ultramontana. De este periódico y de cómo la prensa católica reflejará a partir de los años sesenta las disidencias entre constitucionales y ultramontanos nos ocuparemos en un próximo estudio. Si bien es cierto que este conflicto entre los citados sectores del catolicismo belga ya se hizo notar con fuerza en algunos momentos del unionismo: es el caso de las persecuciones sufridas por el Journal de Flandres.

En 1838, Delebecque, nada más ser nombrado obispo, manifiesta su deseo de que en Gante sólo se edite un periódico de tendencia católica, por lo que intenta la fusión del anterior con el Constitutionnel des Flandres. Al no aceptar los hermanos Vergauwen, propietarios del Journal, las condiciones del obispo -especialmente la existencia de un censor eclesiástico-, se prohibe al clero su lectura, difusión o cualquier colaboración en la redacción. Coinciden en aplaudir esta medida el Rey, el gobierno austríaco, el Papa y el Obispo de Gante, pero, como puntualiza H. Haag, por motivos diferentes: "Leopoldo I y Metternich porque los católicos progresistas -tal era la tendencia del Journal de Flandres- se oponían a su política, y Gregorio XVI y Delebecque porque estimaban que el catolicismo democrático era contrario al bien de la Iglesia"12. Tras las elecciones de junio de 1839, en las que los conservadores se impusieron a la coalición de progresistas y orangistas, la lectura del Journal de Flandres fue de nuevo prohibida a los clérigos, mientras que el episcopado recomienda la lectura del Organe de

9 A. Simon reproduce una carta de Sterckx a Nève, fechada el 18 de diciembre de 1857, en la que, modificando la actitud que describíamos más arriba, el Cardenal se compromete a donar al Journal veinte francos por año Cfr. L'Hypothèse Libérale en Belgique. Documents inédits 1839-1907 (Wetteren: Editions Scaldis, 1956, p Cfr. $L$.
La Un .
La

10 La Union belge era la versión popular del Journal de Bruxelles, y la Gazette de Bruxelles de la Emancipation. 11 Cfr. BRAIVE, G., "Les groupes de presse belges en 1858", en Revue belge de Philologie et d'Histoire, XVL, n1 2, 1967, pp. 408 a 437.

12 Sobre el conflicto que comentamos y las diverdas propuestas de acuerdo de Delebecque, anteriores y posteriores a la prohibición del Journal de Flandres, cfr. HAAG, H., Les origines du catholicisme libéral en pelgique (1789-1839). Universidad de Lovaina, 1950, pp. 234 a 252. 
Flandres, sucesor del Constitutionnel. De este modo, el diario católico progresista fue condenado a la desaparición, que tuvo lugar pocos años después.

Con carácter más anecdótico, cabe destacar que el diario belga más antiguo entre los que se editan actualmente, Le Courrier de l'Escaut, fundado en 1829 por iniciativa de Dumortier y un grupo de católicos de Tournai, destacó por su solitaria defensa del unionismo hasta ya avanzados los años sesenta.

\section{Primeras publicaciones demócratas}

Aunque el Partido Obrero no se organiza en Bélgica hasta 1884, el pensamiento demócrata-socialista se va configurando lentamente desde los primeros momentos de la independencia. Ya entonces, sus líderes, en su mayoría burgueses, promoverán distintas publicaciones para exponer sus planteamientos ideológicos. Asimismo, son frecuentes los panfletos propagandísticos, en los que se defienden la instrucción obligatoria, un sistema impositivo reglado en función de los ingresos..., y el gran caballo de batalla: el sufragio universal. Entre estos folletos destaca El Catecismo del Proletario, de Victor Tedesco.

Durante la etapa unionista es patente el influjo de las doctrinas extranjeras. La primera que arraiga con cierta fuerza entre los intelectuales radicales belgas es la saint-simoniana. Para propagarla, el jefe de la misión belga, Duveyrier, funda en Bruselas, el 29 de mayo de 1831, L'Organisateur belge, que apenas dura cuatro meses.

Entre 1838 y 1848, en sucesivas visitas al país vecino, Victor Considérant intenta difundir los postulados fourieristas en Bélgica. El eco de sus conferencias produjo una crisis en el periódico demócrata más influyente anterior a 1848: Le Débat Social. Así, el 5 de abril de 1846, su primer director, Adolphe Bartels, decide abandonar el semanario por sus divergencias con otros de los fundadores, los hermanos Delhasse, que se habían pronunciado desde las páginas de esta publicación en favor de la nueva doctrina fourierista, difícilmente conciliable con el catolicismo de Bartels.

Bajo la dirección de Felix Delhasse, los planteamientos de Le Débat Social se moderan, hasta el punto de condenar la nacionalización de la industria y contar con colaboradores que defienden el libre cambio. Desde el Congreso liberal de junio de 1846 y hasta las jornadas de febrero del 48, el periódico se convierte en el órgano de la sociedad L'Alliance, que aunaba al sector más progresista del liberalismo, aunque, cuando Jottrand asume la dirección en septiembre de 1847, comienzan a acentuarse de nuevo las demandas de carácter social.

Junto a Le Débat Social hay que destacar en la década de los treinta una hoja flamenca, De Volksvriend, promovida por el obrero Jacques Kats en 1836, así como Le Radical, editado entre abril de 1837 y agosto de 1838, y que contaba con firmas tan notables del pensamiento democrático belga como Lucien Jottrand. 
El ciclo revolucionario de 1848, junto con la abolición del impuesto del timbre el 28 de mayo de ese mismo año, provoca el florecimiento de nuevas publicaciones de tendencia demócrata-socialista, casi todas ellas efímeras y ya no siempre vinculadas, como había ocurrido en los años anteriores, a intelectuales radicales. Al abaratarse el precio de los periódicos y ser posible la venta al número los mismos obreros comenzarán a editar periódicos.

El 26 de abril de 1848 sale a la calle el primer número de La Nation, que será una de las publicaciones políticas más relevantes de la capital belga a mediados de siglo. Ante los problemas económicos que atravesaba, en 1854 los accionistas deciden ceder la propiedad del periódico a su redactor jefe, Louis Labarre, quien, a su vez, conservando la dirección política, cede los asuntos financieros a un amigo demócrata, Stappaerts, cuyas relaciones con el impresor de L'Echo de Bruxelles, M. Nathalis Briavoine, harán que éste último acabe haciéndose con la propiedad de La Nation en enero de 1856. Briavoine modifica la cabecera, que pasa a denominarse Le National y Labarre funda una nueva Nation, que dejará de editarse en agosto del mismo año, como consecuencia de un artículo en el que el mismo Labarre acusaba a la Duquesa de Brabant, esposa del príncipe heredero, de ser un arma del gobierno austríaco en su política de apoyo a las pretensiones imperialistas que sobre Bélgica tenía Napoleón III.

Labarre edita desde diciembre de 1856 un nuevo periódico, Le Drapeau, orientado a la polítia exterior y de tendencia netamente más moderada que $L e$ Prolétaire, que se había convertido en septiembre de 1855, de la mano del comunista libertario Nicolas Coulon, en una de las primeras publicaciones socialistas belgas de cierto eco redactada por obreros.

A pesar de los fracasos electorales de los radicales en las elecciones legislativas de junio 1848, en las elecciones de oficiales de la guardia cívica, celebradas al mes siguiente, y en las comunales de agosto del mismo año, la democracia belga continuó expresando sus ideales a través de órganos como $L a$ Voix du Peuple, cuyo principal redactor era un obrero de nombre Lois y $L a$ Civilisation, editada, como el anterior, en Bruselas, por el fourierista Henri Samuel; Le Peuple, L'Ouvrier y Le Travailleur -surgido a partir de una escisión entre el editor y el impresor del anterior- en Lieja; La Réforme, en Verviers; Le Démocrate, en Charleroi; Artevelde, Vlaenderens Welvaeren, y Broedermin, en Gante; Het Brugesche Vry, en Brujas; y De Vriend des Volks, en Amberes.

Por otro lado, merece mención la Deutche Brüsseler Zeitung, órgano de los socialistas alemanes refugiados en Bélgica, que comienza a publicarse en Bruselas en enero de 1847. Si bien es cierto que este periódico no tuvo mucha influencia en el desarrollo del socialismo belga, conviene recordar, como apunta Louis Bertrand, que se trata de la tercera publicación en importancia de la democracia europea de entonces, tras la Northern Star londinense y La Réforme 
parisina $^{13}$. En la Deutche Brüsseler Zeitung escribieron Marx y Engels hasta su expulsión en marzo de 1848 ante las presiones del gobierno prusiano, que logró el cierre de la Gaceta.

Esta dispersión de los periódicos que acabamos de describir y que contrasta, como hemos visto, con lo que sucedía en el ámbito católico, evidencia la falta de organización del movimiento obrero belga a mediados de siglo.

A modo de conclusión. Los estudios sobre la prensa liberal.

El planteamiento de este artículo exigía un apartado que se ocupase con cierto detalle de la prensa liberal, pero la documentación que hemos consultado al respecto es demasiado incompleta y no nos permite, por el momento, acercarnos al tema con la claridad que el lector merece. No obstante, sí podemos indicar cuáles eran las cabeceras más significativas del ámbito liberal en los últimos años de nuestra etapa de estudio. Así, en 1857 se crea L'Echo du Parlement, promovido por el sector doctrinario del liberalismo, que necesitaba contar con un nuevo órgano de prensa ante el giro progresista que habían experimentado L'Observateur y L'Independence.

También en la línea del sector liberal doctrinario -más concretamente relacionado con la figura de Charles Rogier-, merece especial mención un grupo de prensa, que bajo la forma de una sociedad en comandita se funda en Amberes en 1858: el grupo Outendirck, configurado a partir de la compra del Précurseur, Avenir, De Vlaemsche Stem, Schelde, Toekomst, y Nieuwsblad der provincie Limburg. Todos estos periódicos compartían las informaciones y mostraban ligeras divergencias en la línea editorial. Cabe destacar que L'Avenir era una especie de versión popular del Précurseur, al parecer, como indica G. Braive, una práctica frecuente entonces ${ }^{14}$.

Igualmente inspirado por Frère-Orban, el Journal de Liège permanece, desde finales del siglo XVII y hasta 1870 , en manos de la familia Desoer. Y, aunque en un tono más moderado, los doctrinarios cuentan también en Lieja con La Meuse, fundada en 1855 por un grupo de políticos, industriales y financieros de la zona, y dirigida durante mucho tiempo por Léon de Thier.

A mediados de siglo también habían comenzado a editarse en Bruselas dos periódicos republicanos y profranceses: L'Independence y L'Etoile Belge, éste último propiedad de la familia Orleáns. A ambos haremos referencia en un próximo trabajo por los problemas diplomáticos que generaron en 1870 al hilo de la guerra franco-prusiana.

13 La mayor parte de los datos que recogemos en este epígrafe han sido tomados del detallado relato de Louis Bertrand, Histoire de la Démocratie et du Socialisme en Belgique depuis 1830 (Bruselas: Dechenne et Cía., 2 tomos, 1906-1907), así como del trabajo de R. Demoulin, De la presse radicale aux premiers organe ouvriers (1830-1849) (Bruselas: Bibliothèque de la Révolution de 1848, tomo XXIII, 1966, pp. 191-214). 14 Cfr. BRAIVE, G., op. cit., pp. 408 a 437. 
Por otro lado, queremos apuntar que, si bien es verdad que la prensa decimonónica es una prensa esencialmente política -y como tal en Bélgica se adscribe a las corrientes liberal, católica o socialista-, no es menos cierto que ya a mediados del siglo pasado se vislumbran en este país intentos de configurar grupos de prensa, movidos más por razones económicas que por inquietudes ideológicas. Es el caso de Nathalis Briavoinne, que en 1858 publica dos periódicos de tendencia liberal (el Echo de Bruxelles y el Télégraphe), cuando diez años antes había dirigido el grupo de la Emancipation, portavoz del sector conservador de Félix de Merode. Y ello sin olvidar la operación que ya hemos relatado con respecto a La Nation de Labarre.

Junto al ejemplo de Briavoinne merece mención Adrien Carton de Wiart, quien, tal vez actuando en nombre de un grupo financiero franco-inglés ${ }^{15}$, se hizo en 1858 con la propiedad del Télégraphe, el Observateur, y el Journal de Belgique, e intentó adquirir, aunque sin éxito, la Revue de Namur, el Echo de Bruxelles y la Emancipation.

Finalmente, hemos de indicar que durante esta etapa la prensa flamenca tuvo un carácter marginal, pues las publicaciones en lengua neerlandesa no comienzan a proliferar hasta avanzados los años cuarenta.

15 Sobre los dos casos que comentamos, cfr. BRAIVE, op. cit., pp. 416 a 420 